\title{
CONSTRAINTS ON NEUTRON STAR PARAMETERS FROM BURST OSCILLATION LIGHT CURVES OF THE ACCRETING MILLISECOND PULSAR XTE J1814-338
}

\author{
Sudip Bhattacharyya ${ }^{1}$, Tod E. Strohmayer ${ }^{2}$, M. Coleman Miller ${ }^{1}$, and Craig B. \\ Markwardt ${ }^{1,2}$
}

\begin{abstract}
Detailed modeling of the millisecond brightness oscillations during thermonuclear bursts from low mass X-ray binaries can provide important information about neutron star structure. Until now the implementation of this idea has not been entirely successful, largely because of the negligible harmonic content in burst oscillation lightcurves. However, the recent discovery of non-sinusoidal burst oscillation lightcurves from the accreting millisecond pulsar XTE J1814338 has changed this situation. We, therefore, for the first time, make use of this opportunity to constrain neutron star parameters. In our detailed study of the lightcurves of 22 bursts, we fit the burst oscillation lightcurves with fully general relativistic models that include light-bending and frame-dragging for lightcurve calculation, and compute numerically the structure of neutron stars using realistic equations of state. We find that for our model and parameter grid values, at the $90 \%$ confidence level, $R c^{2} / G M>4.2$ for the neutron star in XTE J1814-338. We also find that the photons from the thermonuclear flash come out through the layers of accreted matter under conditions consistent with Thomson scattering, and show that the secondary companion is a hydrogen burning main sequence star, with possible bloating (probably due to X-ray heating).
\end{abstract}

Subject headings: equation of state - radiative transfer — relativity — stars: neutron - X-rays: binaries - X-rays: bursts

\footnotetext{
${ }^{1}$ Department of Astronomy, University of Maryland at College Park, College Park, MD 20742-2421; sudip@astro.umd.edu, miller@astro.umd.edu

${ }^{2}$ Laboratory for High Energy Astrophysics, Goddard Space Flight Center, NASA, Greenbelt, MD 20771; stroh@clarence.gsfc.nasa.gov, craigm@milkyway.gsfc.nasa.gov
} 


\section{Introduction}

Nearly coherent brightness oscillations have been discovered with the Rossi X-ray Timing Explorer $(R X T E)$ during thermonuclear X-ray bursts from more than a dozen neutron stars in low-mass X-ray binaries (Strohmayer \& Bildsten 2003). The large modulation amplitudes at the onset of bursts, the time evolution of the pulsed amplitude during the rise of bursts (Strohmayer, Zhang, \& Swank 1997), the coherence of the oscillations (Smith, Morgan, \& Bradt 1997; Strohmayer \& Markwardt 1999; Muno et al. 2000), and the long term stability of the oscillation frequencies (Strohmayer et al. 1998) led quickly to an interpretation in terms of a "hot spot" on the surface of the star, so that the observed flux is modulated by rotation (Strohmayer et al. 1996). This interpretation has been strongly supported by observations of the transient accretion-powered pulsars SAX J1808-3658 (Chakrabarty et al. 2003) and XTE J1814-338 (Markwardt, Strohmayer, \& Swank 2003), in which the frequency of burst oscillations is indeed extremely close to the spin frequency as inferred from persistent oscillations.

Early on it was suggested that the intensity profiles of the pulse lightcurves contain valuable information about the stellar mass and radius (and hence about the equation of state of the dense matter in the stellar core), as well as the surface emission properties and system geometry (Strohmayer, Zhang, \& Swank 1997; Miller \& Lamb 1998; Weinberg, Miller, \& Lamb 2001). The reason is that the lightcurves are affected by general relativistic light deflection as well as special relativistic beaming and aberration, which can be significant at the $\sim 0.1-0.2 \mathrm{c}$ linear rotational speeds implied for spin frequencies of a few hundred Hertz. However, much of the required information is encoded in the ratios of pulse amplitudes at different harmonics of the spin frequency (although some constraints can be obtained if the amplitude at the fundamental is particularly high; see Nath, Strohmayer, \& Swank 2002). Until recently, no source had harmonics detected definitively (for upper limits see Muno, Özel, \& Chakrabarty 2002).

This situation has now changed. Analysis of XTE J1814-338, the fifth known accreting millisecond pulsar, shows a clear overtone in the pulse profiles of many individual bursts, with an amplitude that can be more than 0.25 times that of the fundamental (Strohmayer et al. 2003). In addition, the $314 \mathrm{~Hz}$ frequency of the fundamental oscillation is consistent with the spin frequency seen from the persistent pulsations. The existence of harmonics and the confirmation of the basic hot spot picture make this source very promising for detailed analysis and constraints on stellar, geometrical, and emission properties. We note that even more precise information is in principle available from the extremely accurately characterized lightcurves of the persistent pulsations of millisecond pulsars, which have several harmonics (e.g., see Cui, Morgan, \& Titarchuk 1998; Poutanen \& Gierliński 2003). However, 
the spectrum and profiles of pulses during accretion are affected by complicated details of shock physics and Comptonization. In contrast, thermonuclear burst spectra are well fit by blackbodies, without any trace of a high-energy tail related to Comptonization. They are also consistent with coming from very near the stellar surface. For these reasons, the pulses of XTE J1814-338 are most promising for an initial investigation. Combined with analyses of surface spectral line profiles (e.g., from EXO 0748-676; Cottam, Paerels, \& Mendez 2002), thermonuclear X-ray bursts are key sources for constraining the state of matter inside neutron stars.

Here we report our analysis of the RXTE PCA data on 22 thermonuclear bursts from XTE J1814-338. In $\S 2$ we briefly describe our data extraction techniques. In $\S 3$, we discuss our theoretical models, the fitting procedure and the method of calculation of confidence intervals. We discuss our results in $\S 4$. In particular, we show that for the two highdensity equations of state (EOS) that we analyze, there is a preference for comparatively less compact neutron stars. We also show that the emission pattern from points on the surface is constrained moderately strongly and is close to that expected from the law of darkening for passive radiative transport in a Thomson scattering atmosphere (Chandrasekhar 1960). In this paper we use geometric units, i.e., $G=c \equiv 1$.

\section{Data Analysis}

XTE J1814-338 was discovered in the Galactic bulge monitoring campaign carried out with the RXTE PCA (Markwardt \& Swank 2003). The system has a binary orbital period of 4.28 hours and the neutron star has a spin frequency of $314.36 \mathrm{~Hz}$ (Markwardt et al. 2004, in preparation). This is the widest binary among the five known accreting millisecond pulsar

systems. A total of 27 X-ray bursts were observed during the extensive RXTE follow-up of the outburst. A summary of basic burst properties can be found in Strohmayer et al. (2003). For the purposes of our modeling here we wished to produce lightcurves with as high a statistical precision as possible, while still maintaining energy resolved profiles. To do this we elected to phase-align different bursts (while preserving any misalignments among the energy bands). The energy channel boundaries were chosen so that the lightcurves in each band would have roughly similar statistical qualities (ie. similar numbers of total counts). We used the channel boundaries; 0-3, 4-6, 7-10, 11-13 and 14-28 of the "E_125us_64M_0_1s" PCA event mode. This corresponds to energy boundaries of $\approx 2-3.7 \mathrm{keV}, 3.8-5.0 \mathrm{keV}, 5.2$ - $6.6 \mathrm{keV}, 6.8-9.2 \mathrm{keV}$, and $9.4-23 \mathrm{keV}$. To co-add the bursts we found an offset phase for each burst which maximized the $Z_{2}^{2}$ signal when adding the burst in question to the initial, reference burst. This is basically the same procedure described by Strohmayer \& Markwardt 
(1999).

Although the different burst profiles are qualitatively similar, and hence adding them together is reasonable (see Figure 1 for added burst profile), we found that there is a general trend for the harmonic strength to decrease with time. That is, bursts occurring later in the outburst seem to have somewhat smaller ratios of the amplitude of the first overtone compared to that of the fundamental. This suggests that some secular change associated with the accretion rate might be influencing the details of the pulse profiles. Therefore, to allow for such changes while still obtaining good statistics, we added together the bursts in three groups based on their times of occurrence (see Figures 2, 3 and 4 for added burst profiles for three groups). Since the overall structure (ie. the mass and radius) of the neutron star must remain fixed through the outburst, the secular changes in the pulse profile may reflect small changes in the spot size, location or perhaps beaming function, with time. We will say more about this shortly.

\section{Physical Effects and Calculations}

The basic picture we employ is one in which after leaving the photosphere, photons propagate freely in vacuum to the observer. This approach ignores any scattering during the propagation. If there were scattering by a hot plasma near the star, we would expect this to leave its Comptonization imprint on the spectrum as an extended power-law tail. The lack of such a signature (Strohmayer et al. 2003) supports the assumption of free propagation.

Even with this simplification, if the emission pattern on the surface and the angledependence of specific intensity from a given point on the surface are arbitrary, then there are too many free parameters and no meaningful constraints are possible. Therefore, to make progress in modeling, we adopt the following assumptions:

- The surface consists of a background of uniform intensity and spectrum, plus a single hot spot that is a filled circle that emits uniformly. This is the most popular simple emitting system for burst oscillations. In a future paper we will consider models with two hot spots.

- For $R / M<3.52$ and for Schwarzschild spacetime, an emitted photon is deflected by more than $180^{\circ}$ (Pechenick, Ftaclas, \& Cohen 1983). Therefore, to simplify numerical techniques by ensuring that no emitted photon is deflected by more than $180^{\circ}$, the minimum value of $R / M$ we consider is 3.6.

With these assumptions, we calculate lightcurves by tracing rays backwards, i.e., from 
the observer to the stellar surface. The paths of these rays, and their specific intensities, carry the imprint of several physical effects. Using an approach similar to that of Özel \& Psaltis (2003), we consider: (1) Doppler boosts, (2) special relativistic beaming, (3) gravitational redshift, (4) light-bending, and (5) frame-dragging. We do not include the effects of spininduced stellar oblateness. These effects are only second order in the stellar rotation and are thus small compared to other uncertainties. For example, for all our EOS models, the polar to equatorial radii ratio of the star is greater than 0.98 (for stellar mass $M \geq 1.4 M_{\odot}$ and observed stellar spin frequency $\nu_{*} \sim 314 \mathrm{~Hz}$ ). We describe tests of our raytracing and lightcurve codes in the Appendix.

To make our model lightcurves realistic, we calculate the relation between $M$ and the stellar radius $R$, as well as $a / M$ (where, $a$ is the stellar angular momentum per unit mass) for a given $M$ and $\nu_{*}(\sim 314 \mathrm{~Hz}$ for XTE J1814-338), with a specified EOS model. We do this by computing numerically the stable structure of a rapidly spinning neutron star, using the formalism given by Cook, Shapiro, \& Teukolsky (1994) (for a detailed description, see also Bhattacharyya et al. 2000; Bhattacharyya 2002). For a particular neutron star EOS model and assumed values for the stellar mass and spin rate, we solve Einstein's field equations and the equation of hydrostatic equilibrium self-consistently to obtain other stellar structure parameters (radius, angular momentum, etc.). We use these output parameters in our timing calculations.

In our detailed lightcurve calculation, we have five input parameters, for a given EOS model and the known value of $\nu_{*}$. In our study, the structure of the star is fixed by one unknown parameter, which we choose to be (1) the dimensionless stellar radius to mass ratio $(R / M)$. The hot spot is specified by two parameters: (2) the $\theta$-position $\theta_{\mathrm{c}}$ of the center of the spot, and (3) the angular radius $\Delta \theta$ of the spot. The emission from a single point on the spot, as measured in the corotating frame, is characterized by (4) a parameter $n$ that gives a measure of the beaming in the emitter's frame (corotating with the star), where the specific intensity as a function of the angle $\psi$ (in the emitter's frame) from the surface normal is $I(\psi) \propto \cos ^{n}(\psi)$. Finally, we have (5) the inclination angle $i$ of the observer as measured from the upper rotational pole. It is to be noted, that at the initial phase of our model calculation, we consider that the hot spot emits as a blackbody (as burst spectra can be fitted well by blackbodies) with a temperature $k T=2 \mathrm{keV}$. However, later we allow the source spectrum to deviate from a blackbody (for discussion about the spectrum and the justification of the assumed temperature, see later in this section).

In our work, we consider two representative EOS models: A18 and A18+ $\delta v+$ UIX (Akmal, Pandharipande, \& Ravenhall 1998). The first one is the Argonne $v_{18}$ model (Wiringa, Stoks, \& Schiavilla 1995) of two-nucleon-interaction. This model fits the Nijmegen database 
(Stoks et al. 1993) with $\chi^{2} / N_{\text {data }} \sim 1$, and hence is called "modern". The model A $18+\delta v+$ UIX considers two additional physical effects: the three-nucleon-interaction (Urbana IX (UIX) model; Pudliner et al. 1995) and the effect of relativistic boost corrections to the A18 interaction. The EOS model A18 is soft, i.e., the maximum non-rotating mass it supports is small $\left(\sim 1.67 M_{\odot}\right)$. On the other hand, the EOS model A18 $+\delta v+\mathrm{UIX}$ is hard (maximum supported non-rotating mass is $\sim 2.2 M_{\odot}$ ). Figure 2 shows the stable stellar mass vs. radius curves for these EOS models (for stellar spin frequency $\sim 314 \mathrm{~Hz}$ ). Although, there are many other EOS models available in the literature, our chosen models span a representative range.

In order to compute the energy dependent flux, we trace back the paths of the photons from the observer to the hot spot, using the Kerr spacetime. We solve the geodesic equations numerically using a fifth order Runge-Kutta method with adaptive stepsize control. The accuracy of the results is tested and described in the appendix. Our ray-tracing method is similar to that of Bhattacharyya, Bhattacharya, \& Thampan (2001), except that here we use the Kerr spacetime, instead of the correct spacetime around a rapidly rotating neutron star, for the sake of simplicity. It is unlikely that the errors introduced by this approximation will have any detectable effect on the lightcurves for the stellar spin frequency we consider (i.e., $\sim 314 \mathrm{~Hz}$ ). Our method has two major differences from the approach of, e.g., Braje, Romani, \& Rauch (2000). (1) We solve the geodesic equations of motion of a photon from the observer to the surface of the neutron star, instead of from the surface to the observer. (2) Instead of doing elliptic integral reduction of the geodesics (as was done by Braje et al. 2000), we solve the equations of motion numerically, for easier generalization to numerical spacetimes.

The ray-tracing enables us to get the boundary of the image of the source at the observer's sky. We then calculate the observed flux by integrating the observed energy dependent specific intensity inside the boundary of this image (for a detailed description, see Bhattacharyya et al. 2001). The model lightcurve is calculated by repeating the same procedure for many spots at different $\phi$-positions (but the same $\theta$-position) on the surface of the star. The actual phase points of the lightcurve are calculated from these $\phi$-positions, the stellar spin frequency, and the time delay consideration. The time delay is because photons emitted at different points on the stellar surface take different times to reach the observer.

In this paper, we compare our models with the data of 22 bursts. Based on burst occurrence time and the harmonic strength of the lightcurves, as described above, we added all the bursts in three groups; bursts 1-8; 9-16; and 17-22. We phase-align and stack the bursts within each group, and therefore effectively analyze three characteristic sets of pulse profiles. We used five energy bands for each group. The energy ranges are given in $\S 2$ above. 
We compare our models directly with the data. To do this, we compute a model lightcurve of intensity and spectrum versus pulse phase. In this initial step we consider only the lightcurve of the hot spot, not any background emission. We fold the model spectrum through the RXTE response matrix to get counts per channel as a function of rotational phase. For each channel range, we then add a phase-independent background (which is a free parameter) and normalize it so that the total number of counts in that channel range, summed over all phases, is the same as the number of counts in that channel range in the observed lightcurve. Our final step is to shift the entire lightcurve in phase (by same amount for all the channel bands) to maximize the match with the observed lightcurve, as determined by a $\chi^{2}$ statistic.

The procedure of adding backgrounds and normalizing counts independently in each of the channel ranges has two implications. First, the independent backgrounds mean that we leave the unpulsed spectrum unconstrained. Second, the independent normalizations mean that although we calculate the initial spectrum and lightcurve of the hot spot assuming a $k T=2 \mathrm{keV}$ blackbody, renormalization allows the effective spectrum to deviate from blackbody. Therefore, we preserve the rms variability of a blackbody with, e.g., Doppler shifts, but not the whole spectrum. Indeed, in reality we do not expect the temperature to be constant throughout a hot spot; for example, if the hot spot is related to a magnetic pole, it might be hotter in the center than at the edges. In addition, the pulse profiles extracted from the observations incorporate data over a period of several tens of seconds, during which the hot spot is likely to cool significantly. Thus, the time-averaged spectrum will not truly be a blackbody, hence we let the spectrum be adjusted by allowing independent normalizations.

The initially chosen hot spot temperature of $2 \mathrm{keV}$ is justified for the following reasons: (1) It is expected to be close to the average blackbody temperature of the hotspot for most of the bursts (see Kuulkers et al. 2003 for blackbody temperature variation during burst evolution for several sources), and (2) The likelihood distributions (see next two paragraphs for description) for the parameters are similar, even for widely different initially chosen hot spot temperatures.

Using the above procedure of adding backgrounds, normalizing, and shifting phases, we find the best $\chi^{2}$ value for many different combinations of parameters. To be systematic, we consider combinations of parameters on a grid (for the values of each parameter, see Table 1). We also use the Markov Chain Monte Carlo (MCMC) method to ensure that the grid method does not miss any significantly low $\chi^{2}$ values. The number of counts per phase bin in each channel range is large enough that Gaussian statistics apply, hence the likelihood is proportional to $\exp \left[-\chi^{2} / 2\right]$. We apply the physical constraint that the value of each parameter is same for all the channel ranges for a given burst group (some parameters, 
for example, the size and the location of the hot spot may change from one burst group to another). Therefore, for each parameter combination, the likelihood is proportional to $\exp \left[-\Sigma \chi^{2} / 2\right]$, where the sum is over all the channel ranges.

Once the likelihoods are computed for each combination of parameters, we produce likelihoods for each parameter individually via the process of marginalization. In this process, let the posterior probability density over the full set of model parameters $\theta_{1} \ldots \theta_{n}$ be $p\left(\theta_{1} \ldots \theta_{n}\right)$. If we are interested in the credible region for a single parameter $\theta_{k}$, then we integrate, or marginalize, this probability distribution over the "nuisance" parameters $\theta_{1} \ldots \theta_{k-1}$ and $\theta_{k+1} \ldots \theta_{n}$ :

$$
q\left(\theta_{k}\right)=\int d \theta_{1} \ldots d \theta_{k-1} d \theta_{k+1} \ldots d \theta_{n} p\left(\theta_{1} \ldots \theta_{n}\right) .
$$

For our purposes we assume that each combination of parameters has the same a priori probability, hence the posterior probability is simply proportional to the likelihood. For Gaussian distribution of likelihoods of a parameter, this method is equivalent to the standard frequentist procedure. However, for non-Gaussian distribution, this Bayesian method is more general.

We find that the posterior probability distributions are not Gaussian for most of our parameters. Therefore, although there is a unique maximum likelihood value for any parameter, credible regions cannot be quoted uniquely. For example, one gets a different value if one assumes a symmetric distribution than if one looks for the smallest region containing a certain total probability. We have adopted a variant of the latter procedure. Using linear interpolations of the probability between grid points for a given parameter, we compute the smallest region that includes the maximum likelihood point and contains $90 \%$ of the total probability. For parameters expected to remain unchanged between bursts (specifically, $R / M$ and the observer inclination $i$, and also the beaming parameter $n$ ) we combine data

from all bursts. For the other parameters, we calculate likelihoods separately for each of the three burst groups.

\section{Results and Conclusions}

As discussed in $\S 3$, we perform comparisons using two EOS models. Figure 6 shows the goodness of fit for a certain burst group and channel range, and for a representative combination of parameter values. The $\chi^{2}$ value for this example comes out to be $\sim 25.5$, for the number of degrees of freedom equal to 24 . This goodness of fit suggests that the overall model framework is a reasonable representation of the data.

In Figure 7 , we plot the likelihoods with the parameter $R / M$, for two EOS models, 
using the data from all 22 bursts. While we can not determine an upper limit of $R / M$, lower limits (4.7 for A18+ $\delta v+$ UIX and 4.2 for A18) of $90 \%$ confidence regions (given in Table 2) can be computed. We do not consider $R / M$ values greater than 6.0, as for these values, the stellar mass is too small $\left(<1.07 M_{\odot}\right.$ for A18 and $<1.29 M_{\odot}$ for $\left.\mathrm{A} 18+\delta v+\mathrm{UIX}\right)$ to be realistic for an accreting neutron star. A simple extrapolation of the likelihood curves in Figure 7 shows that the probability of $R / M<3.6$ is very small. However, for $R / M<3.52$ and for Schwarzschild spacetime, photons from a single point on the stellar surface may reach the observer by multiple paths. As a result, the corresponding lightcurves may be qualitatively different from the ones we calculate. Therefore, we can say that one interpretation of the data is that $R / M$ is greater than 4.2 with $90 \%$ confidence, but we can not completely exclude the possibility of $R / M<3.52$.

Figure 8 displays the likelihood distribution with the observer's inclination angle $i$. This figure shows that $i>22^{\circ}$ with very high probability. Here, as well as for the likelihood calculation of other parameters, we focus our fitting procedure on the range $20^{\circ}-50^{\circ}$ for $i$. This is because, with a smaller number of grid points for other parameters, our fitting for inclination angle values in the range $5^{\circ}-90^{\circ}$ shows that the likelihood values for $i<20^{\circ}$ are very small, and the inclusion of inclination angles greater than $50^{\circ}$ produces only minor changes to our results. However, constraints on inclination angle by observations in other wavelengths would help us constrain other parameters more effectively. Presently, M. Krauss et al. (2004, in preparation) are working on this using optical data.

We keep the beaming parameter $n$ (defined in $\S 3$ ) fixed in all three burst groups in our statistical procedure. This is because the thermonuclear flash is expected to occur at the bottom of a pile of accreted matter. Therefore, although the original emission may be isotropic, what comes out through the layers of accreted matter should be beamed. As the optical depth of these layers is expected to be very high, we expect the law of darkening (and hence the value of $n$ ) to be the same for all the bursts.

The likelihood distributions for $n$ for two EOS models are shown in Figure 9. For both EOS models, the beaming parameter $n$ peaks at $n=0.75$. This figure shows that $n$ is well constrained on lower side, and the lower limit of the $90 \%$ confidence regions (for both the EOS model) is 0.55 (given in Table 2). The law of darkening for semi-infinite plane-parallel layers with a constant net flux and Thomson scattering is given in Chandrasekhar (1960). In Figure 10, we compare this law of darkening $I(\psi)$ with our emission function $\cos ^{n}(\psi)$. We find that, for $n=0.55$ (where likelihood value is $\sim 15-20 \%$ of the peak likelihood value), our emission function matches with $I(\psi)$ quite well, except for nearly tangential emission (for which the emitted flux is small). The matching is also reasonably good for the most probable value $n=0.75$. Therefore, the data are consistent with Thomson scattering 
through an optically thick layer (probably in the accumulated accreted matter) of thermal electrons.

In Table 3, we show the $90 \%$ confidence intervals for the polar angle of the center of the hot spot $\left(\theta_{c}\right)$ for both the EOS models. Here we give the results for the three burst groups separately, as the values of this parameter may change from one group to another. The union of $90 \%$ confidence intervals for all burst groups and EOS models is $60^{\circ}-139^{\circ}$. Assuming that the hot spot is at the magnetic pole, this implies an angle of $40^{\circ}-90^{\circ}$ between the spin axis and the magnetic axis. The peak of the likelihood distribution for $\theta_{\mathrm{c}}$ always occurs around the interval $90^{\circ}-110^{\circ}$ (see Figures 11, $12 \& 13$ ), hence the magnetic pole may be close to the rotational equator.

Table 4 displays the likelihood values for the angular radius $(\Delta \theta)$ of the hot spot for different burst groups and EOS models. For the burst group $1-8, \Delta \theta$ is comparatively better constrained, and a smaller spot $\left(\Delta \theta \sim 5^{\circ}-25^{\circ}\right)$ is likely. For the other two burst groups, comparatively larger spots are probable.

A direct comparison of the likelihoods of two EOS models shows that the difference is not enough to constrain EOS models by this direct method.

The pulsar mass function and orbital period for this source are $0.002016 M_{\odot}$ and 4.27 hours respectively (Markwardt, Strohmayer, \& Swank 2003). Hence, a reasonable range of neutron star mass $\left(1.4 M_{\odot}-2.0 M_{\odot}\right)$ and our $i>20^{\circ}$ inclination angle constraint imply a companion mass of $0.17 M_{\odot}-0.72 M_{\odot}$. For this range of masses and for the observed orbital period, the companion can be neither a helium main sequence star nor a degenerate star (Bhattacharya \& van den Heuvel 1991). It is also too large to be a brown dwarf (as was predicted for another accreting millisecond pulsar system SAX J1808.4-3658; Bildsten \& Chakrabarty 2001). The most probable option is that it is either a hydrogen burning main sequence star, or an evolved (sub-)giant star. However, the companion mass is too small for it to have evolved off the main sequence (Böhm-Vitense 1992). Therefore, the most likely option is a hydrogen main sequence star. This is also seen in Figure 14. If the companion is a normal hydrogen burning main sequence star (as shown in Figure 14), its maximum possible mass is $\sim 0.48 M_{\odot}$. This is because, the radius of the companion can not be smaller than that of a hydrogen main sequence star (for a given mass), but it can be bigger, because it can be bloated due to X-ray heating by the neutron star and/or the accretion disk. This upper limit of companion mass corresponds to a lower limit of observer's inclination angle $24^{\circ}$ (using the pulsar mass function value and a lower limit of neutron star mass $1.4 M_{\odot}$ ). Interestingly, this lower limit of inclination angle is close to that found by our lightcurve fitting. However, if the mass fraction of hydrogen in the hydrogen burning main sequence companion star is very low, then it may have a higher mass than $0.48 M_{\odot}$ (see Rappaport \& 
Joss 1984$)$. If we consider the lower limit $\left(0.17 M_{\odot}\right)$ of the companion mass, then its radius is about $98 \%$ bigger than the normal radius (Bhattacharya \& van den Heuvel 1991).

In conclusion, the significant overtones observed in the pulse profiles of burst brightness oscillations from XTE J1814-338 open up new possibilities for constraints on neutron star structure as well as on source geometry and emission properties. In a subsequent paper, we

will study the analysis possible with future large-area detectors, in particular whether EOS models may be constrained strongly based on their likelihoods.

We thank Arun V. Thampan for providing the rapidly spinning neutron star structure calculation code, and Geoff Ravenhall for supplying the tabulations of nucleonic equations of state. We also thank Deepto Chakrabarty and Miriam Krauss for notifying us about their unpublished work. We acknowledge John Miller, Didier Barret, and Biswajit Paul for reading the paper and sending their comments. Many of the results in this paper were obtained using the Beowulf cluster of the department of astronomy, University of Maryland (courtesy of Derek C. Richardson). This work was supported in part by NSF grant AST 0098436.

\section{REFERENCES}

Akmal, A., Pandharipande, V.R., \& Ravenhall, D.G. 1998, Phys. Rev. C, 58, 1804.

Bhattacharya, D., \& van den Heuvel, E.P.J. 1991, Physics Reports, 203, 1.

Bhattacharyya, S. 2002, A\&A, 383, 524.

Bhattacharyya, S., Bhattacharya, D., \& Thampan, A.V. 2001, MNRAS, 325, 989.

Bhattacharyya, S., Thampan, A.V., Misra, R., \& Datta, B. 2000, ApJ, 542, 473.

Bildsten, L., \& Chakrabarty, D. 2001, ApJ, 557, 292.

Böhm-Vitense, E. 1992, Introduction to stellar astrophysics: vol. 3, Cambridge University Press.

Braje, T.M., Romani, R.W., \& Rauch, K.P. 2000, ApJ, 531, 447.

Chakrabarty, D. et al. 2003, Nature, 424, 42.

Chandrasekhar, S. 1960, Radiative Transfer, Dover Publications.

Cook, G.B., Shapiro, S.L., \& Teukolsky, S.A. 1994, ApJ, 424, 823. 
Cottam, J., Paerels, F., \& Mendez, M. 2002, Nature, 420, 51.

Cui, W., Morgan, E.H., \& Titarchuk, L.G. 1998, ApJ, 504, L27.

Kuulkers, E., den Hartog, P.R., in’t Zand, J.J.M., Verbunt, F.W.M., Harris, W.E., \& Cocchi, M. 2003, A\&A, 399, 663.

Markwardt, C.B., Strohmayer, T.E., \& Swank, J.H. 2003, ATEL, 164.

Markwardt, C.B., \& Swank, J.H. 2003, IAUC, 8144.

Markwardt, C.B., Swank, J.H., Strohmayer, T.E., in 't Zand, J.J.M., \& Marshall, F.E. 2002, ApJ, 575, L21.

Miller, M.C., \& Lamb, F.K. 1998, ApJ, 499, L37.

Muno, M.P., Fox, D.W., Morgan, E.H. \& Bildsten, L. 2000, ApJ, 542, 1016.

Muno, M.P., Özel, F., \& Chakrabarty, D. 2002, ApJ, 581, 550.

Nath, N.R., Strohmayer, T.E., \& Swank, J.H. 2002, ApJ, 564, 353.

Özel, F., \& Psaltis, D. 2003, ApJ, 582, L31

Pechenick, K.R., Ftaclas, C., \& Cohen, J.M. 1983, ApJ, 274, 846.

Poutanen, J., \& Gierliński, M. 2003, MNRAS, 343, 1301.

Pudliner, B.S., Pandharipande, V.R., Carlson, J., \& Wiringa, R.B. 1995, Phys. Rev. Lett., $74,4396$.

Rappaport, S., \& Joss, P.C. 1984, ApJ, 283, 232.

Smith, D., Morgan, E.H. \& Bradt, H.V. 1997, ApJ, 479, L137.

Stoks, V.G.J., Klomp, R.A.M., Rentmeester, M.C.M., \& de Swart, J.J. 1993, Phys. Rev. C, 48,792 .

Strohmayer, T.E. et al. 1996, ApJ, 469, L9.

Strohmayer, T.E. et al. 1998, ApJ, 503, L147.

Strohmayer, T.E., \& Bildsten, L. 2003, in Compact Stellar X-ray Sources, Eds. W.H.G. Lewin and M. van der Klis, (Cambridge University Press: Cambridge), (astro-ph/0301544).

Strohmayer, T.E., \& Markwardt, C.B. 1999, ApJ, 516, L81. 
Strohmayer, T.E., Markwardt, C.B., Swank, J.H., \& in 't Zand, J. 2003, ApJ, 596, L67.

Strohmayer, T.E., Zhang, W., \& Swank, J.H. 1997, ApJ, 487, L77.

Verbunt, F. 1990, in Neutron Stars and Their Birth Events, Ed. W. Kundt, (Kluwer Academic Publishers, Dordrecht).

Weinberg, N., Miller, M.C., \& Lamb, D.Q. 2001, ApJ, 546, 1098.

Wiringa, R.B., Stoks, V.G.J., \& Schiavilla, R. 1995, Phys. Rev. C, 51, 38.

\section{Appendix}

We use three codes in series to calculate the $\chi^{2}$ values. These codes have been checked in different ways. Here we mention a few of them. The ray-tracing code traces back the paths of photons from the observer to the surface of the star, and calculates different parameter values at the footprints of the photons on the surface. For a photon that is emitted from the surface tangentially (i.e., the one with maximum angular momentum), one can calculate the amount of angular deflection at infinity (for the Schwarzschild spacetime). The comparison between these values with the outputs from our code give satisfactory results. For example, when $R / M=4.2$, the total angle traveled by a photon emitted tangentially at the surface is $145^{\circ} .8$, and our code yields $145^{\circ} .1$. The code also calculates the total redshift with typically

less than $0.01 \%$ error. However, a photon travelling directly over a pole of the star gives a few degrees of error in the $\theta$-position of its emission point. But the error in this single ray does not introduce significant error in our final results.

For both the Schwarzschild and Kerr metrics, the value of $L=-p_{\phi} / p_{t}$ can be derived analytically at the observer's position and at the surface of the star. Here $p_{\phi}$ and $-p_{t}$ are photon's $\phi$-angular momentum and energy respectively. At the observer's position

$$
L=-b \sin i \sin \alpha
$$

where, $b$ and $\alpha$ are plane polar coordinates in observer's sky, and $i$ is the observer's inclination angle. At the surface of the star,

$$
L=\frac{g_{\phi \phi}^{1 / 2} \cos \xi}{\sqrt{\frac{g_{\phi t}^{2}}{4 g_{\phi \phi}}-g_{t t}}-\frac{g_{\phi t}}{2 g_{\phi \phi}^{1 / 2}} \cos \xi},
$$

This preprint was prepared with the AAS IATEX macros v5.2. 
where, $\xi$ is the emission angle with $\phi$-direction, and $g_{\mu \nu}$ 's are the metric coefficients of a metric

$$
d S^{2}=g_{t t} d t^{2}+g_{r r} d r^{2}+g_{\theta \theta} d \theta^{2}+g_{\phi \phi} d \phi^{2}+g_{\phi t} d \phi d t
$$

As $L$ remains constant along the photon's path, the values of $L$ from equations (2) and (3) should be the same. We find that with our ray-tracing code the maximum difference in these two values is $\sim 1 \%$.

The lightcurve calculation code computes the energy dependent observed flux from different hotspots on the stellar surface. This is done by the integration of observed specific intensity over the image of the hotspot at the observer's sky. As the image of the hotspot is of irregular shape, this integration is done using a Monte Carlo method. We ensure that different random seed values and different number of points (used for Monte Carlo integration) give a stable result. For example, for parameter combinations giving $\chi^{2} /$ dof $\sim 1$, differences in $\chi^{2}$ s are less than $0.5 \%$. For larger $\chi^{2}$ values, these differences are more, but still very low, and these have little effect because it is the lower $\chi^{2}$ values that dominate the likelihoods. These differences in $\chi^{2} \mathrm{~s}$ appear to be more or less random, hence the differences in total $\chi^{2} \mathrm{~s}$ (addition for different channel ranges and burst groups) are also small. The integrated area is also verified to be correct (with typically less than $0.1 \%$ error) even for a small spot (for example, with $5^{\circ}$ angular radius) with its center at the same $\phi$ and $\theta$ values as of the observer.

Finally, the comparison code compares the theoretical lightcurves with the observed data, and computes the $\chi^{2}$ values. We check this code in different ways. For example, we consider different maximum background values and different steps in background values to ensure a stable result (maximum $\sim 0.03 \%$ difference in $\chi^{2}$ values). We also use synthetic data to confirm that the known parameters are obtained to accuracies compatible with our uncertainty region for the real data. 


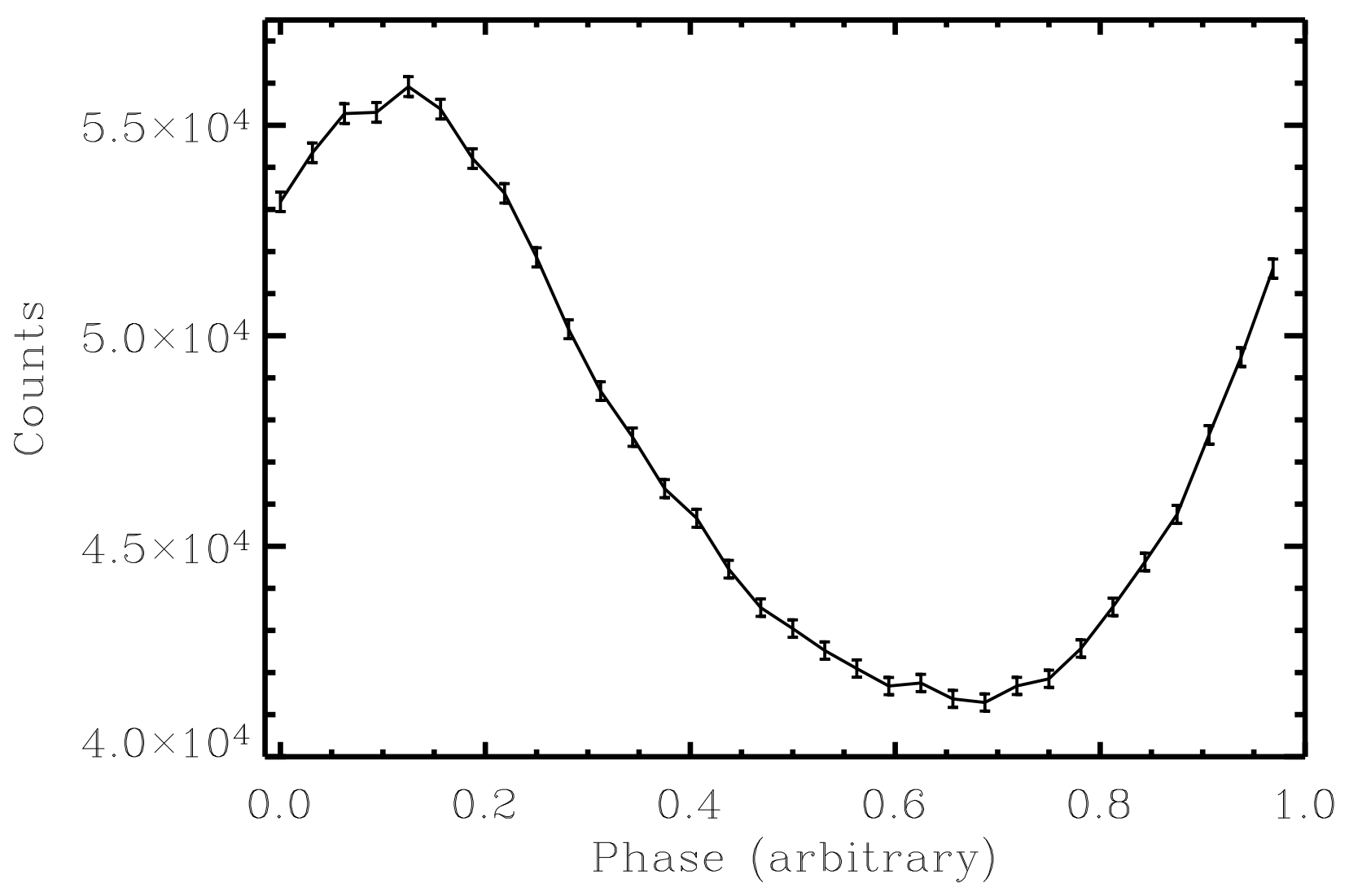

Fig. 1. - The fully added observational lightcurve (22 bursts and $0-28$ channels). 


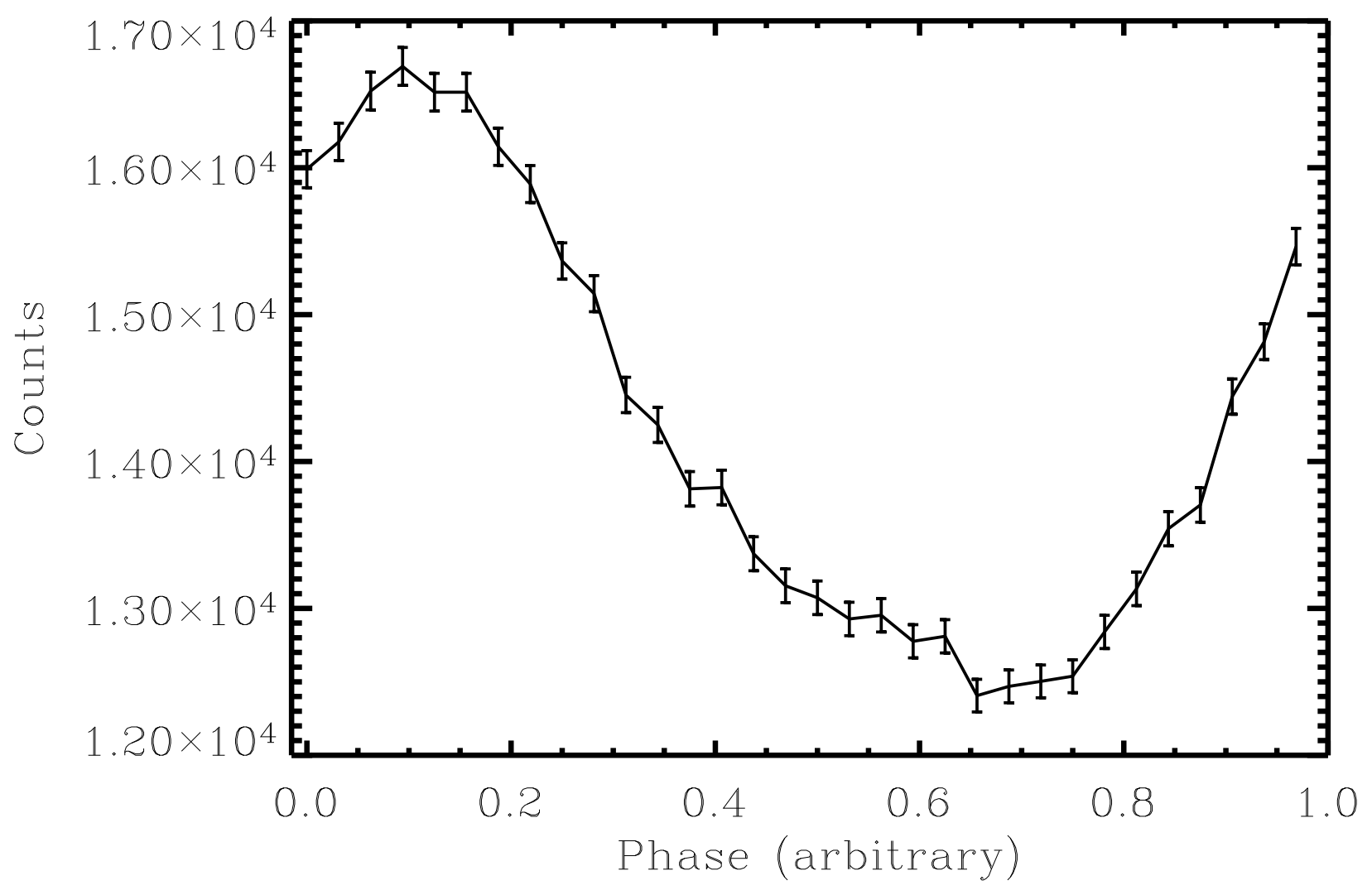

Fig. 2.- The observational lightcurve (bursts: $1-8$ and $0-28$ channels). 


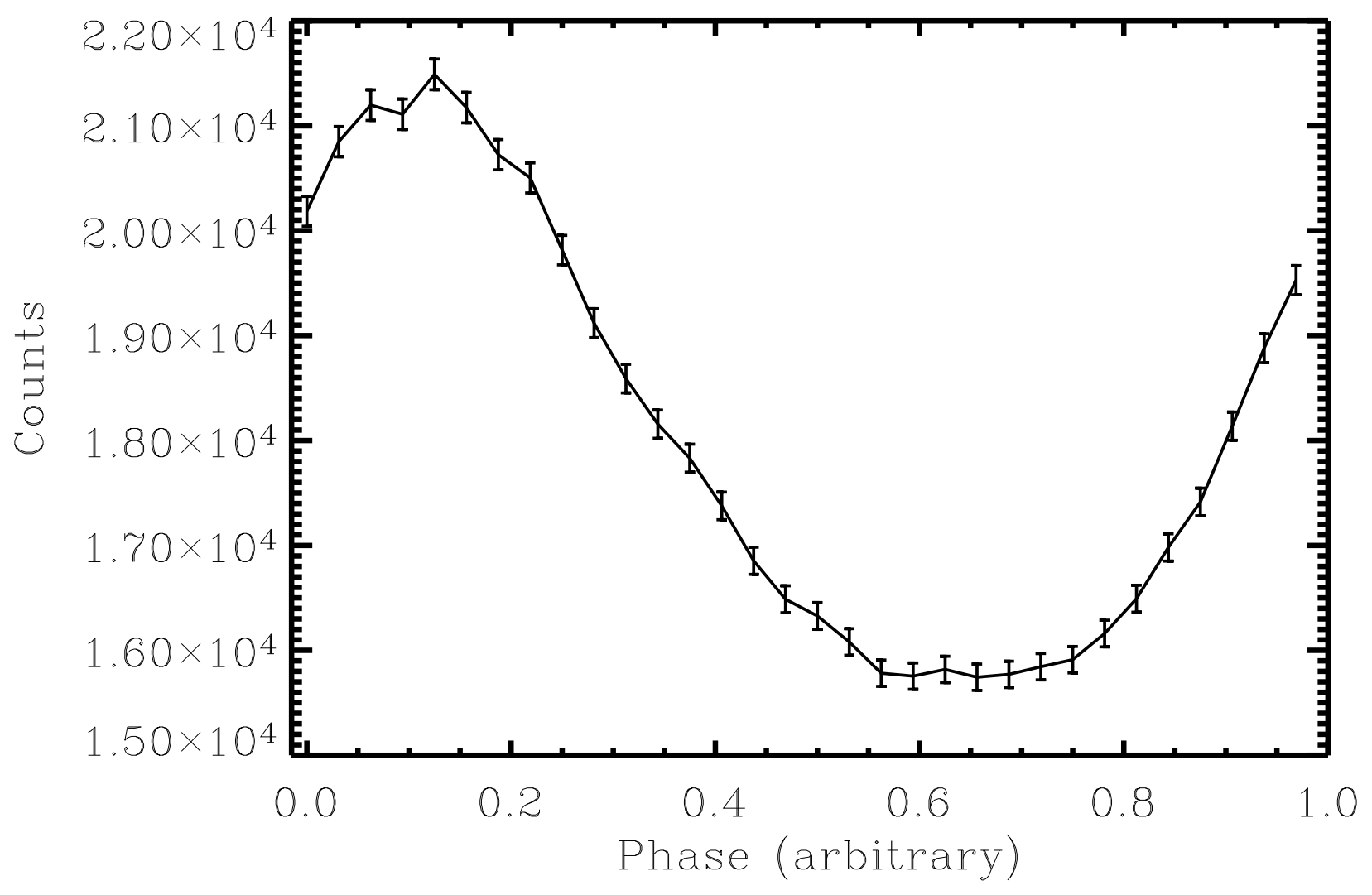

Fig. 3.- The observational lightcurve (bursts: $9-16$ and $0-28$ channels). 


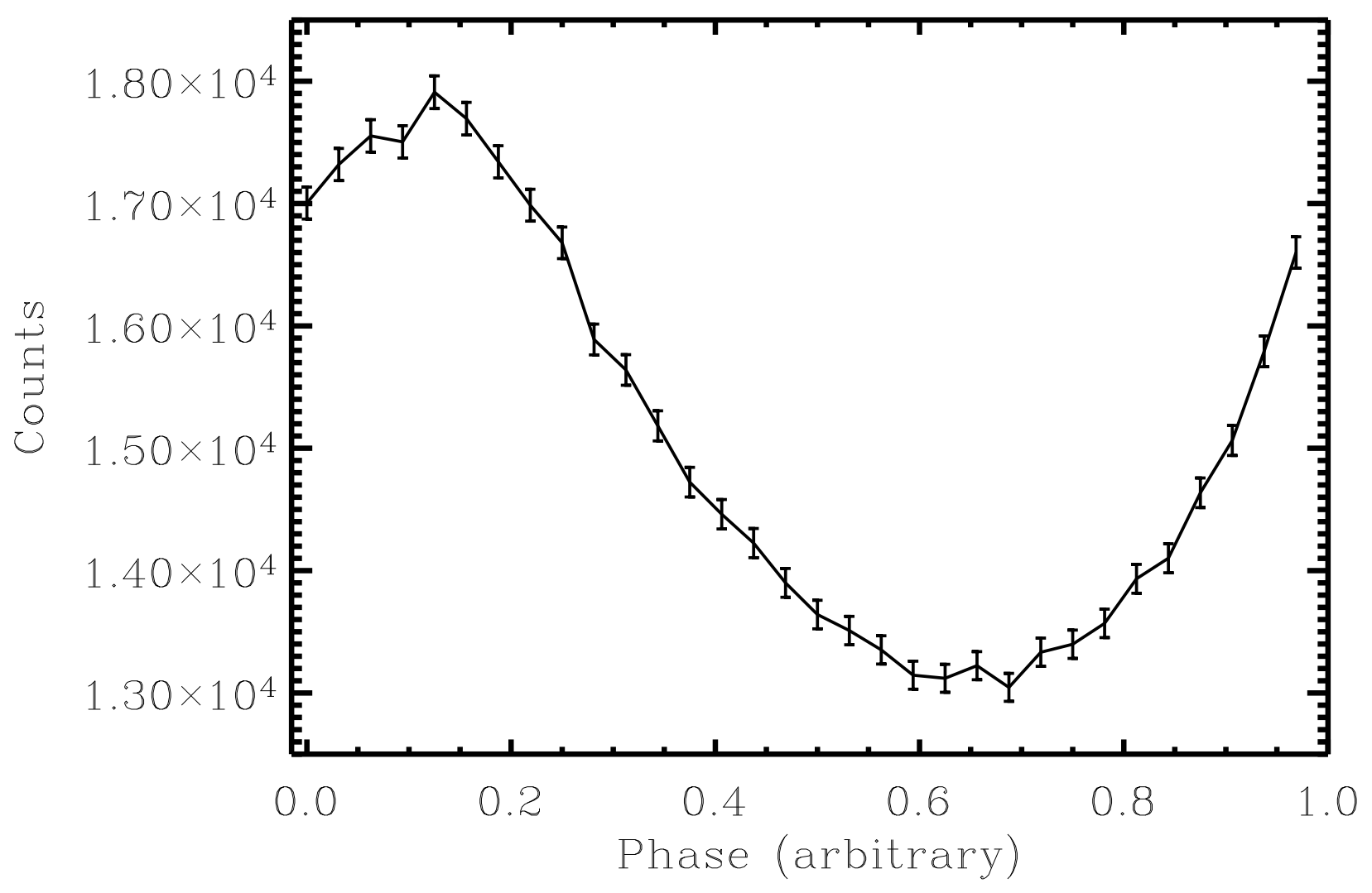

Fig. 4.- The observational lightcurve (bursts: $17-22$ and $0-28$ channels). 


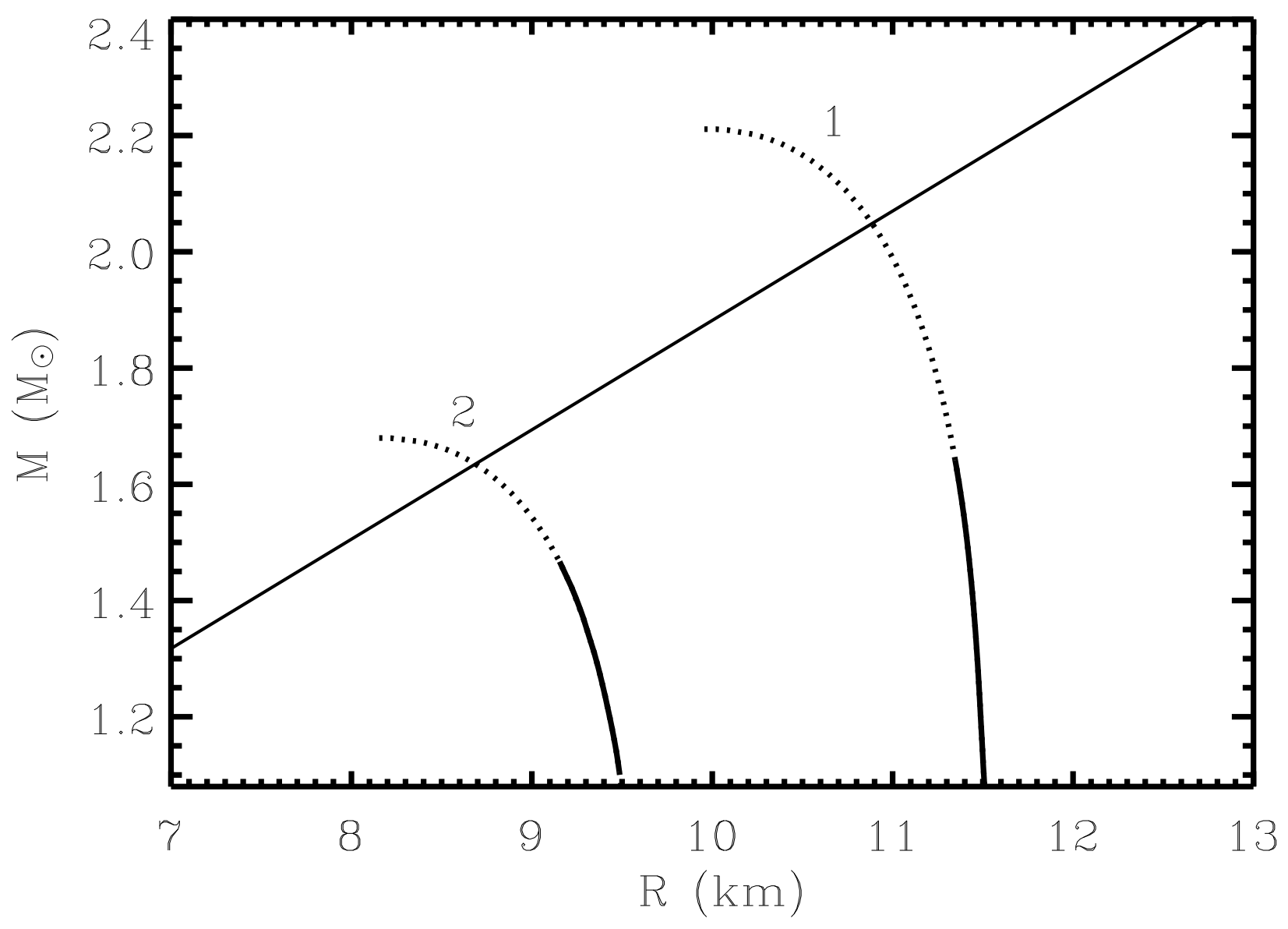

Fig. 5.- Mass vs. radius diagrams: Curve 1 is for the EOS model A18+ $\delta v+$ UIX and curve 2 is for the EOS model A18. These diagrams are for stable stellar configurations, spinning with a frequency $\sim 314 \mathrm{~Hz}$. The solid parts of the curves (correspond to higher $R / M$ sides of the vertical lines in Figure 7) are the allowed regions with $90 \%$ confidence interval of $R / M$. These regions constrain the mass vs. radius plane for the neutron star in XTE J1814-338. The solid oblique line corresponds to $R / M=3.6$. In this work, our results are for the $M-R$ space below this line. 


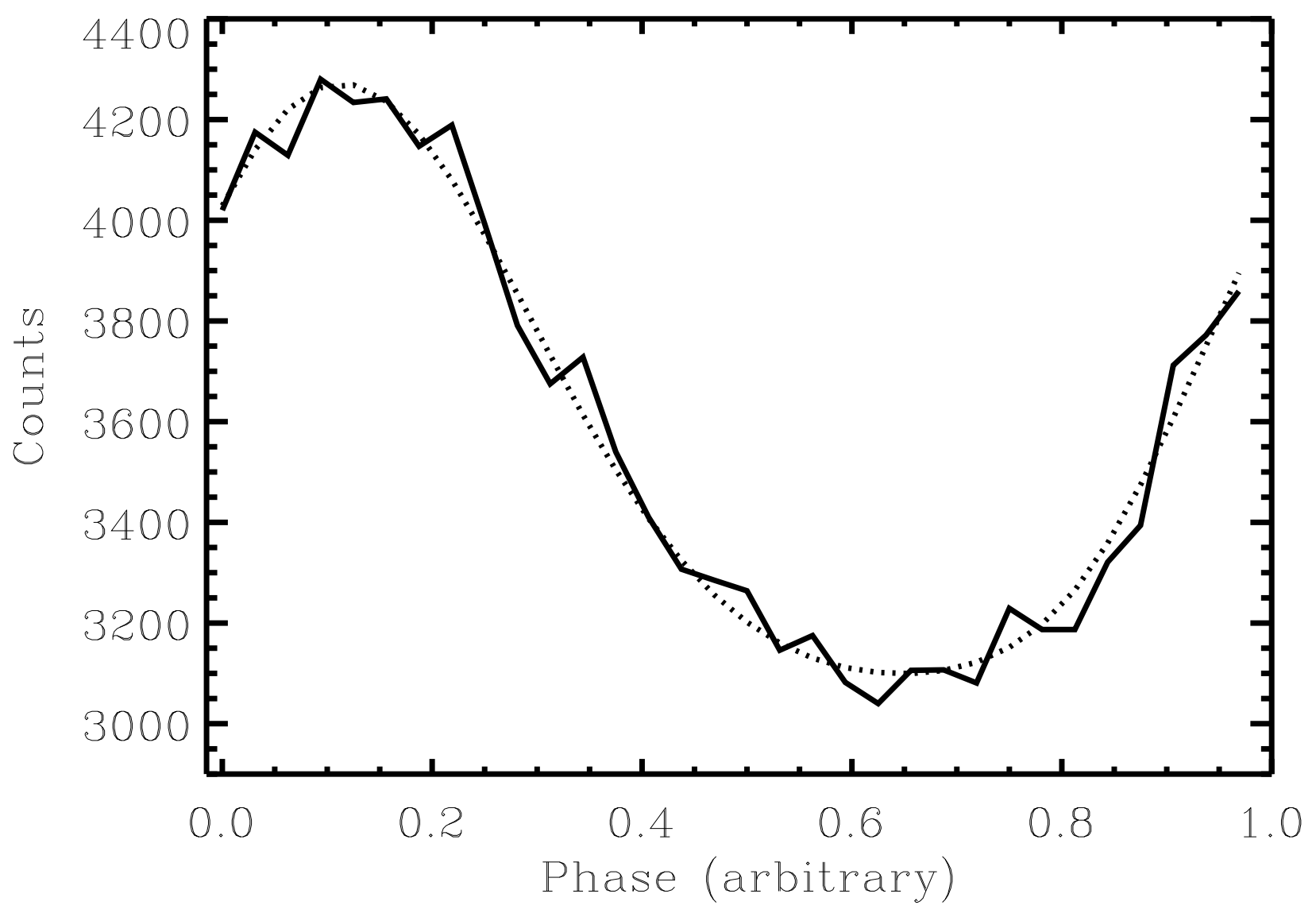

Fig. 6.- An example fit to the data. Stacked data (solid curve) for bursts $9-16$ (channel range: 7 - 10) from XTE J1814-338, versus a model (dotted curve) in which $R / M=4.9$, $i=36^{\circ}, \theta_{\mathrm{c}}=110^{\circ}$, the hot spot has an angular radius of $45^{\circ}$, and $n=0.8$ (see text for description of parameters). Here we have used the EOS model A18+ $\delta v+$ UIX. In this fitting we get a $\chi^{2}$ value $\sim 25.5$, for the number of degrees of freedom equal to 24 . 


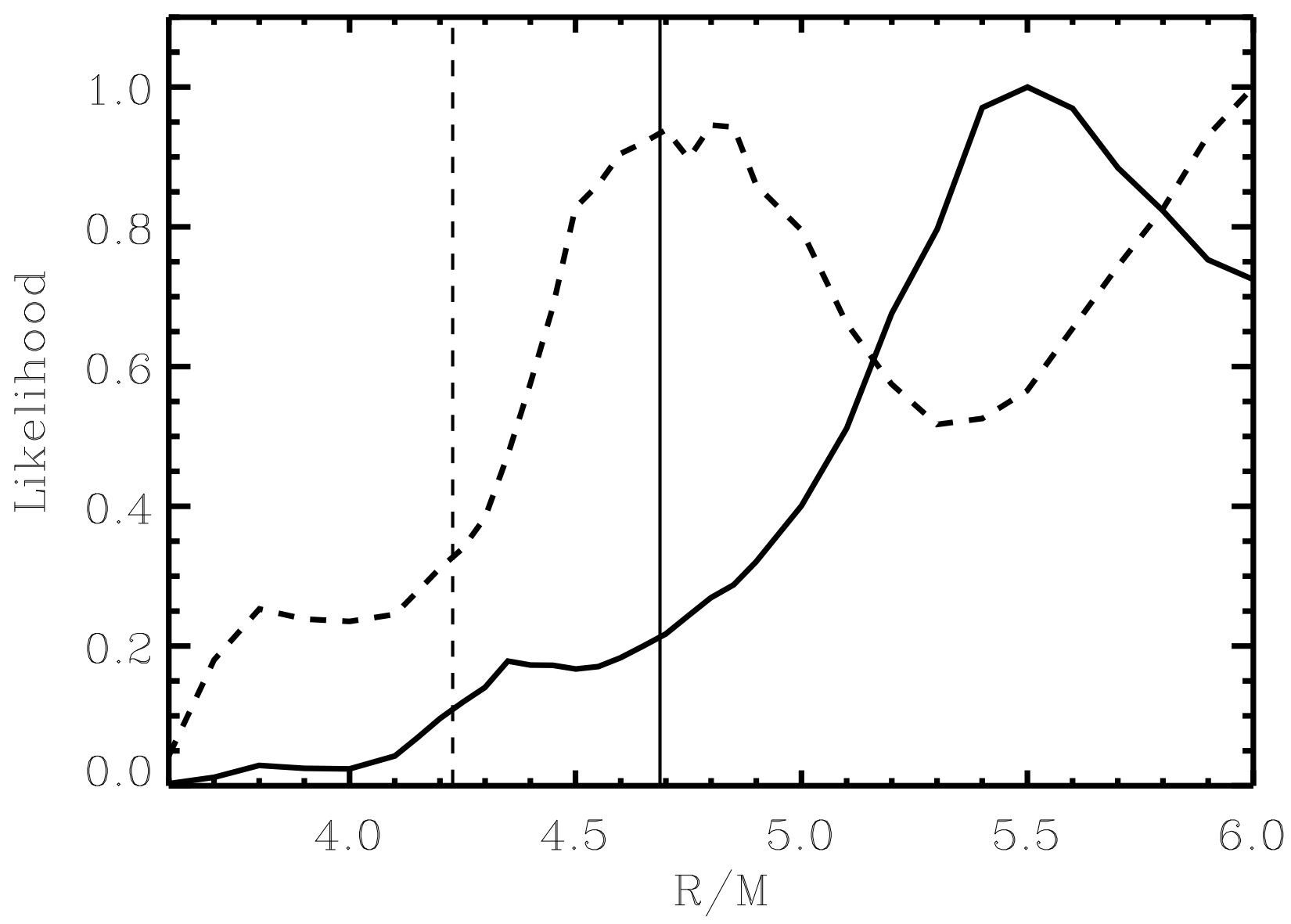

Fig. 7.- Likelihood distribution of stellar radius to mass ratio $R / M$, using data from all 22 bursts. The solid curve is for the EOS model A18+ $\delta v+$ UIX, and the dashed curve is for the EOS model A18. The solid vertical line gives the lower limit of the $90 \%$ confidence region for the EOS model A18+ $\delta v+$ UIX, and the dashed vertical line gives that for A18. 


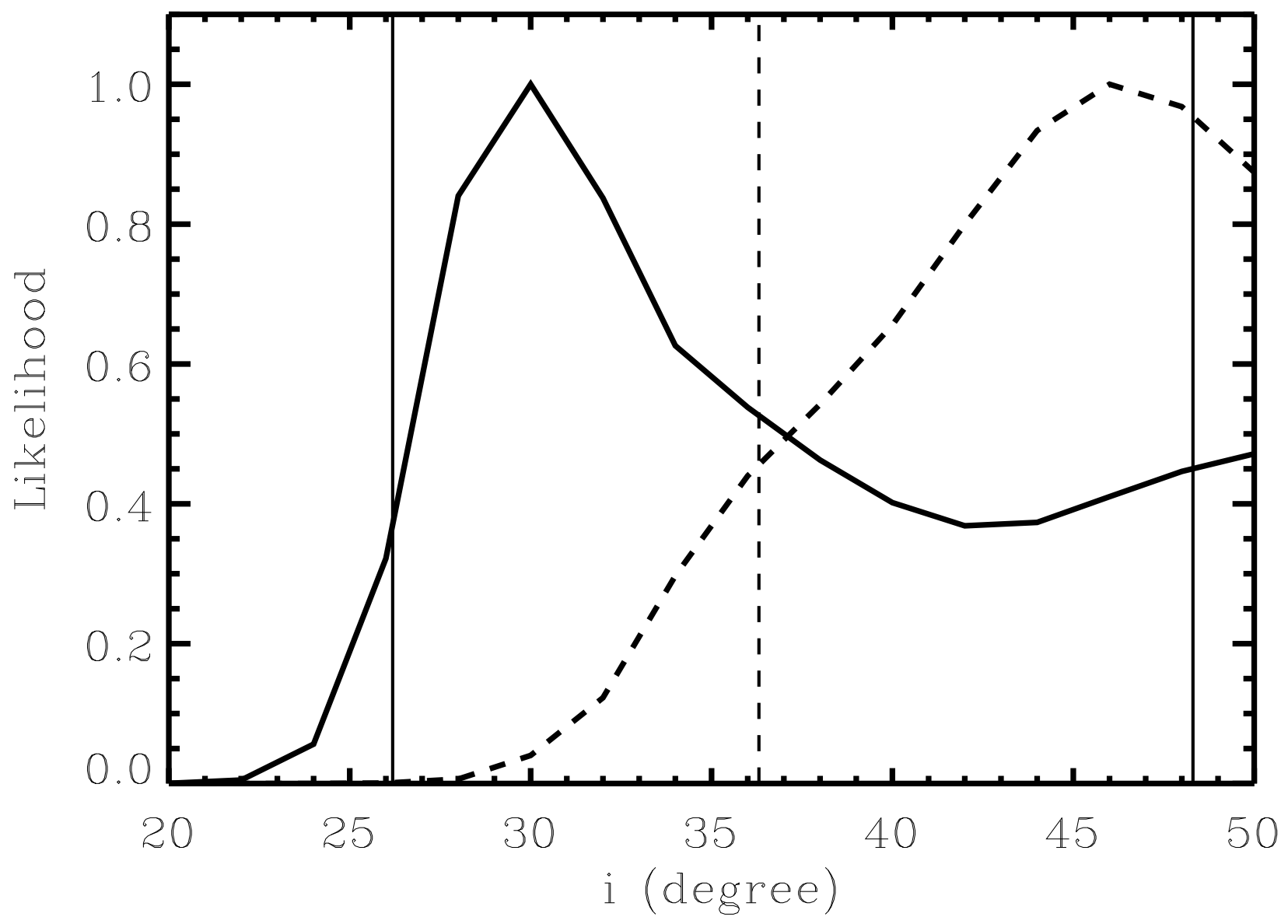

Fig. 8.- Likelihood distribution of observer's inclination angle $i$, using data from all 22 bursts. Solid curve is for the EOS model A18+ $\delta v+$ UIX, and the dashed curve is for the EOS model A18. The solid vertical lines give $90 \%$ confidence interval for the EOS model A18+ $\delta v+$ UIX, while the dashed vertical line gives the lower limit of $90 \%$ confidence region for A18. This figure demonstrates that a value of $i$ less than $22^{\circ}$ is highly improbable. 


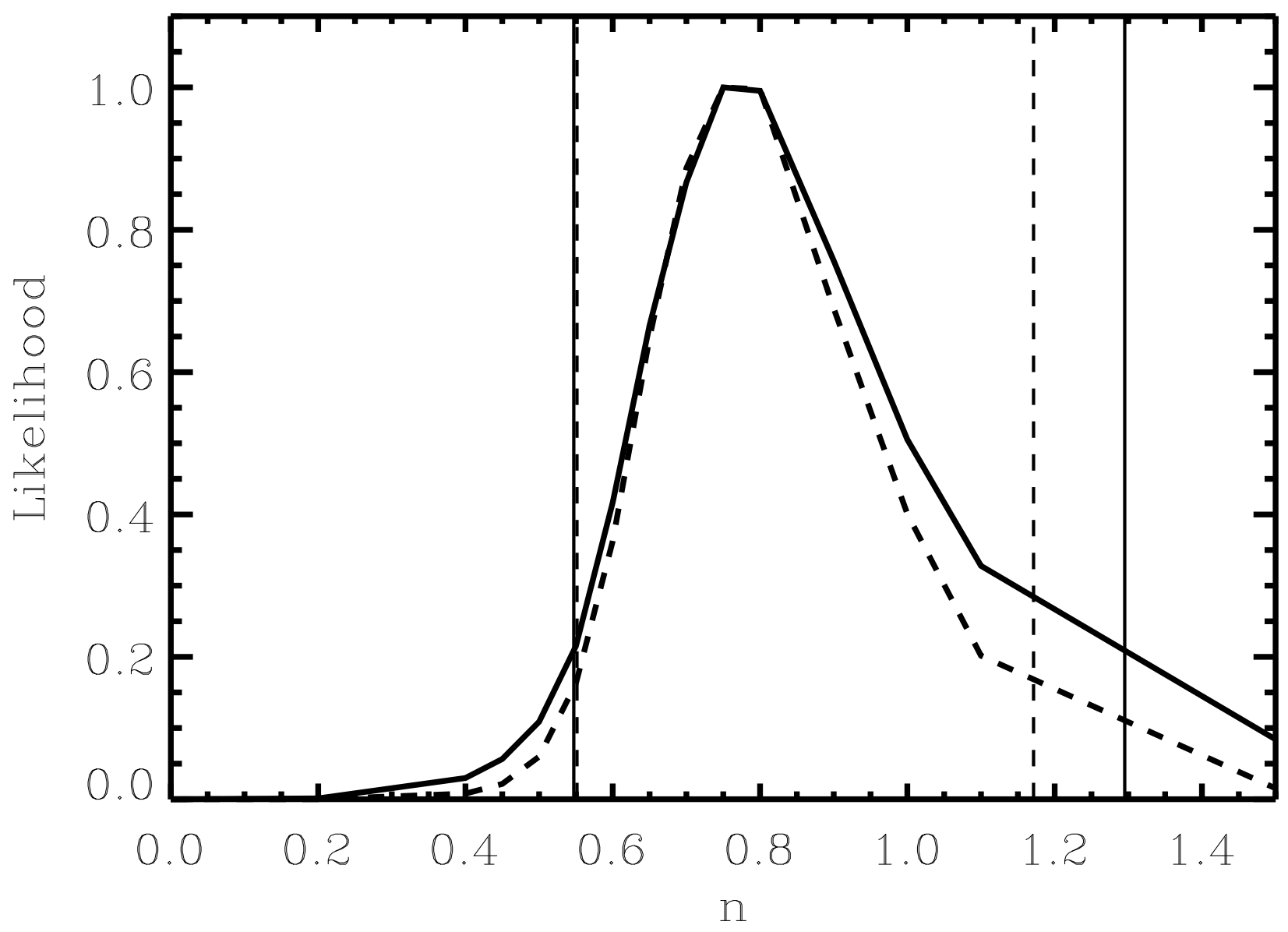

Fig. 9.- Likelihood distribution of the beaming parameter $n$ (defined in $\S 3$ ), using data from all 22 bursts. The solid curve is for the EOS model A18 $+\delta v+$ UIX, and the dashed curve is for the EOS model A18. The solid vertical lines give $90 \%$ confidence interval for the EOS model A18+ $\delta v+$ UIX, while the dashed vertical lines give that for A18. This figure demonstrates that a value of $n$ less than 0.2 is highly improbable. 


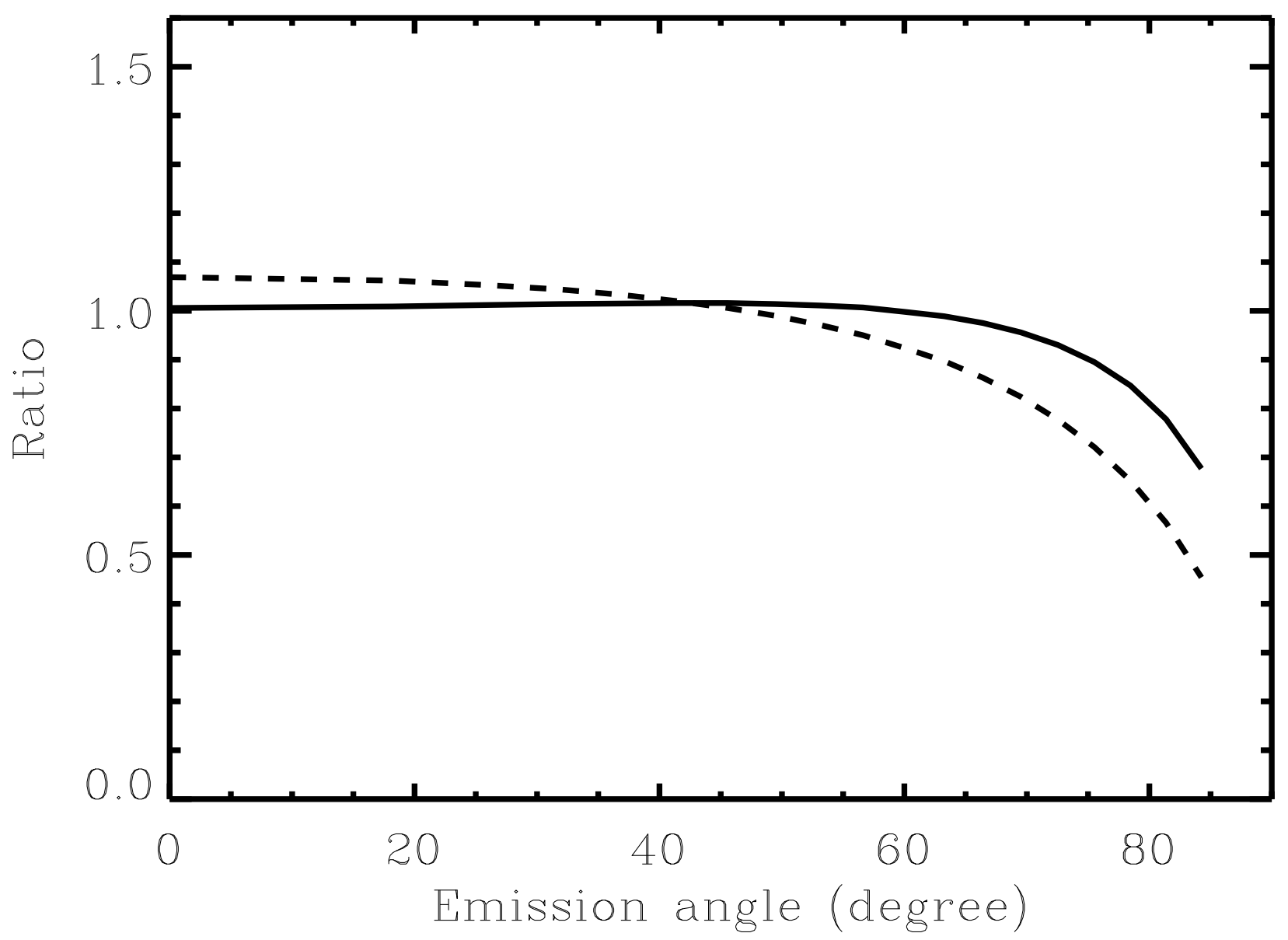

Fig. 10.- Comparison between $I(\psi)$ (Law of darkening; Chandrasekhar 1960) and our emission function $\left(\cos ^{n} \psi\right)$ : Both the functions give the angular distribution of specific intensity in the emitter's frame (corotating with the star). Here the independent parameter is the emission angle $(\psi)$ (measured from surface normal) in the emitter's frame, and the depen-

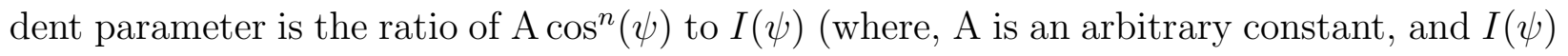
is the law of darkening for the radiative transfer in a semi-infinite plane-parallel atmosphere with a constant net flux and Thomson scattering). The solid curve is for $n=0.55$, and the dashed curve is for $n=0.75$. For the former one, $I(\psi)$ is well fitted by our emission function, except for very high emission angle, for which the emitted flux is small. 


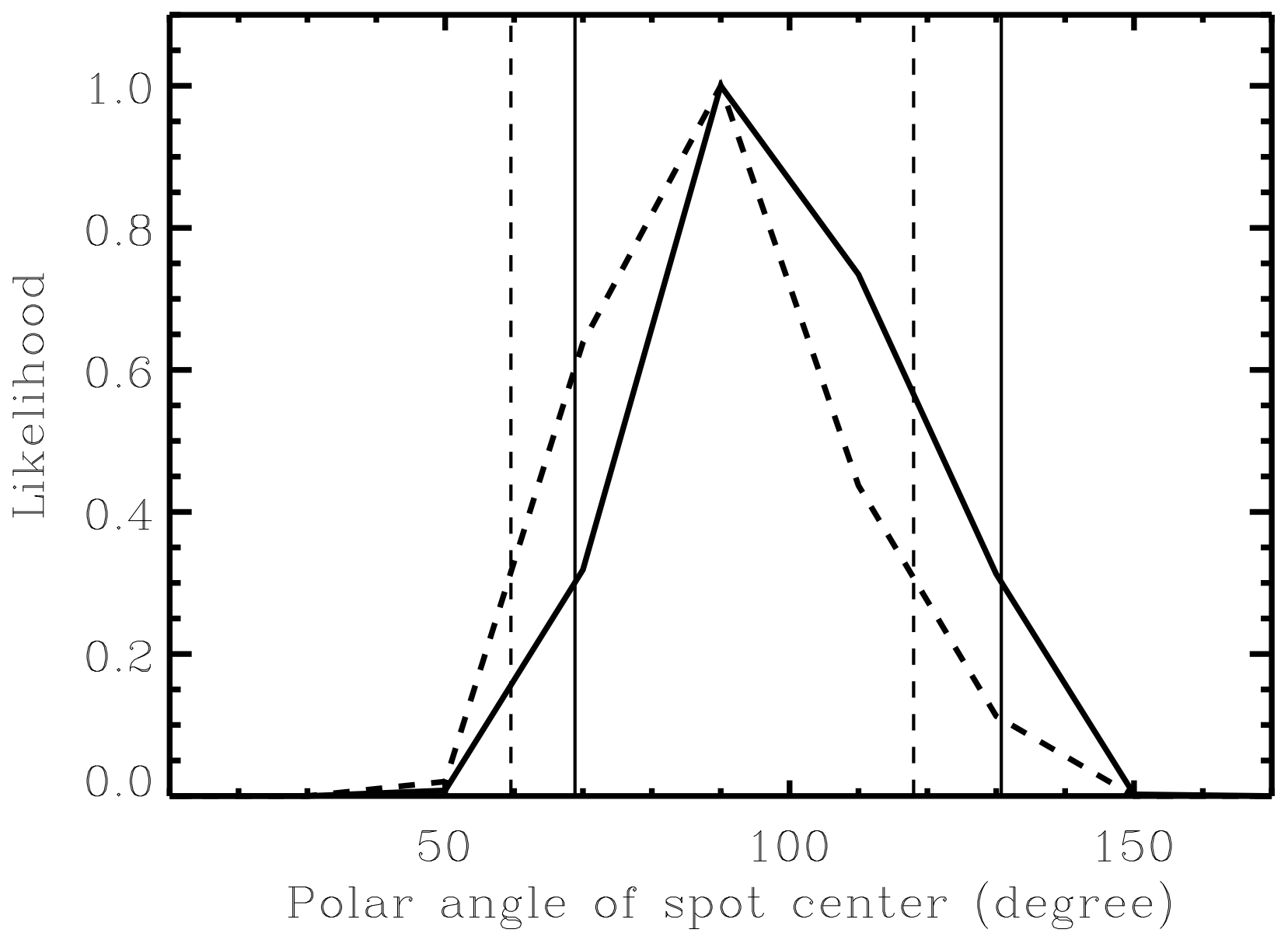

Fig. 11. - Likelihood distribution of the polar angle of the center of the spot $\left(\theta_{c}\right)$ for bursts $1-8$. Solid curve is for the EOS model A18+ $\delta v+$ UIX, and the dashed curve is for the EOS model A18. The solid vertical lines give $90 \%$ confidence interval for the EOS model A18 $+\delta v+$ UIX, while the dashed vertical lines give that for A18. This figure demonstrates that a value of $\theta_{c}$ less than $50^{\circ}$ or greater than $150^{\circ}$ is highly improbable for bursts $1-8$. 


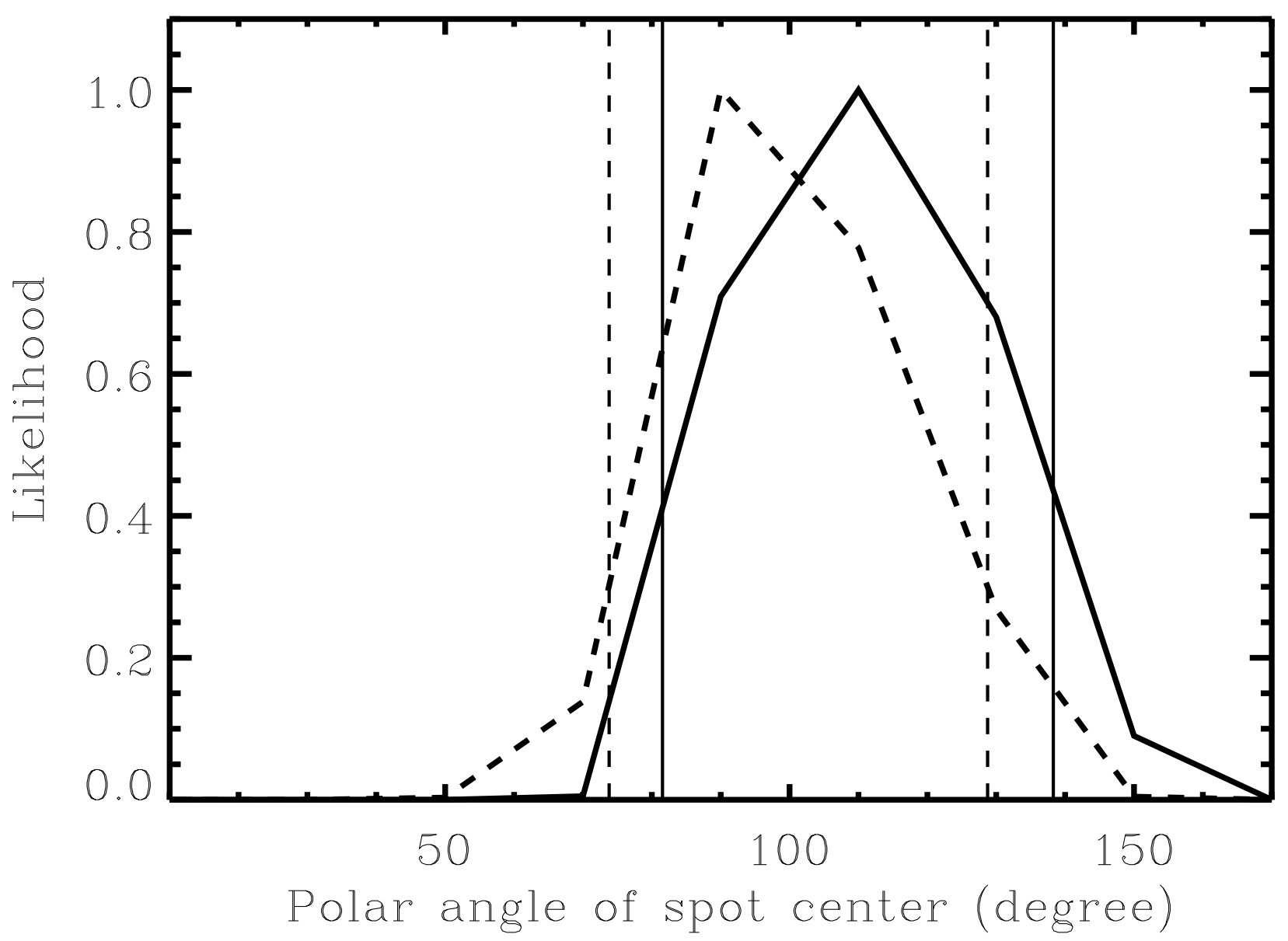

Fig. 12.- Likelihood distribution of the polar angle of the center of the spot $\left(\theta_{c}\right)$ for bursts $9-16$. Meanings of all the curves and lines are same as in Figure 11. This figure demonstrates that a value of $\theta_{c}$ less than $50^{\circ}$ is highly improbable for bursts $9-16$. 


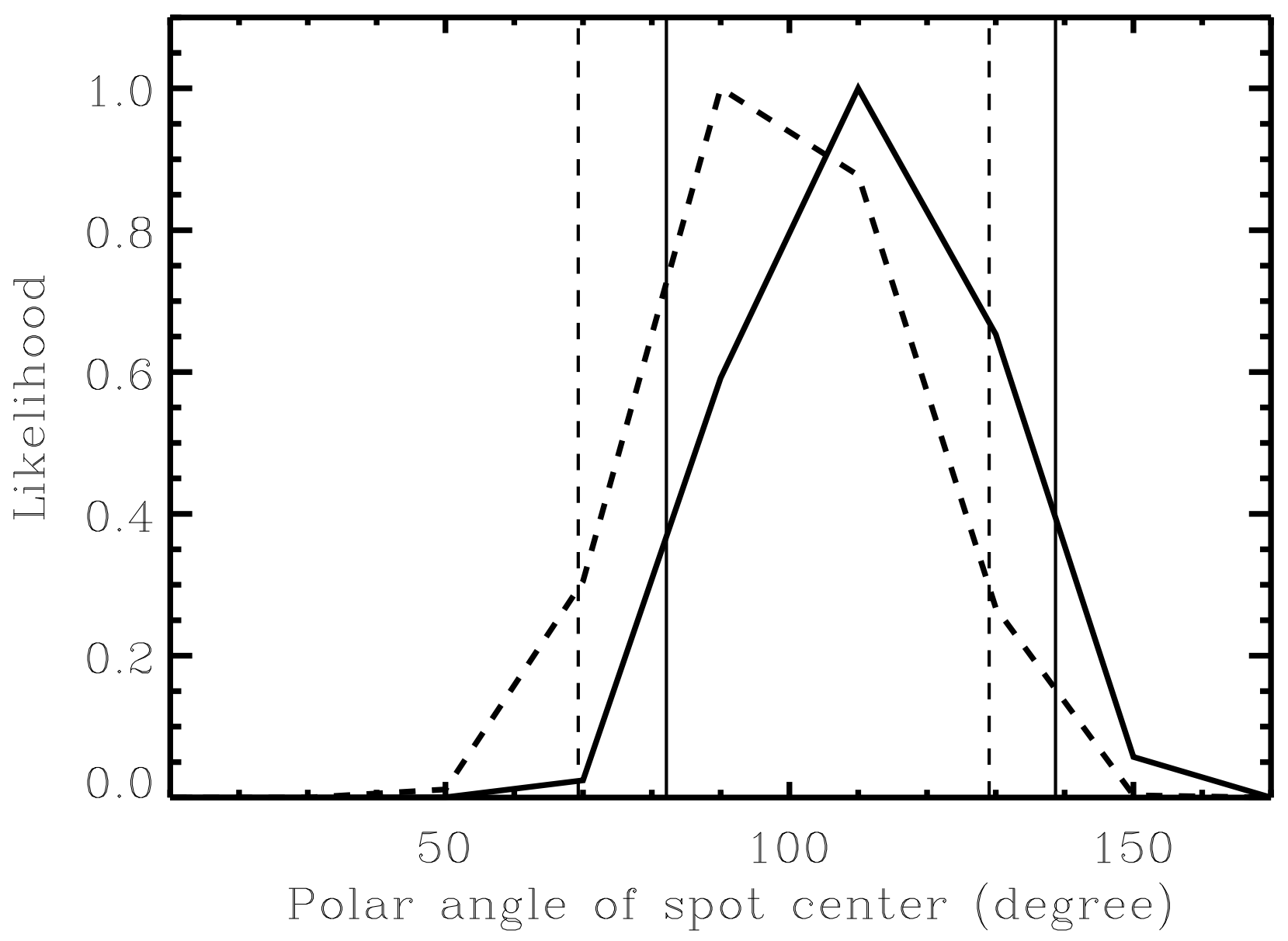

Fig. 13. - Likelihood distribution of the polar angle of the center of the spot $\left(\theta_{c}\right)$ for bursts $17-22$. Meanings of all the curves and lines are same as in Figure 11. This figure demonstrates that a value of $\theta_{c}$ less than $50^{\circ}$ is highly improbable for bursts $17-22$. 


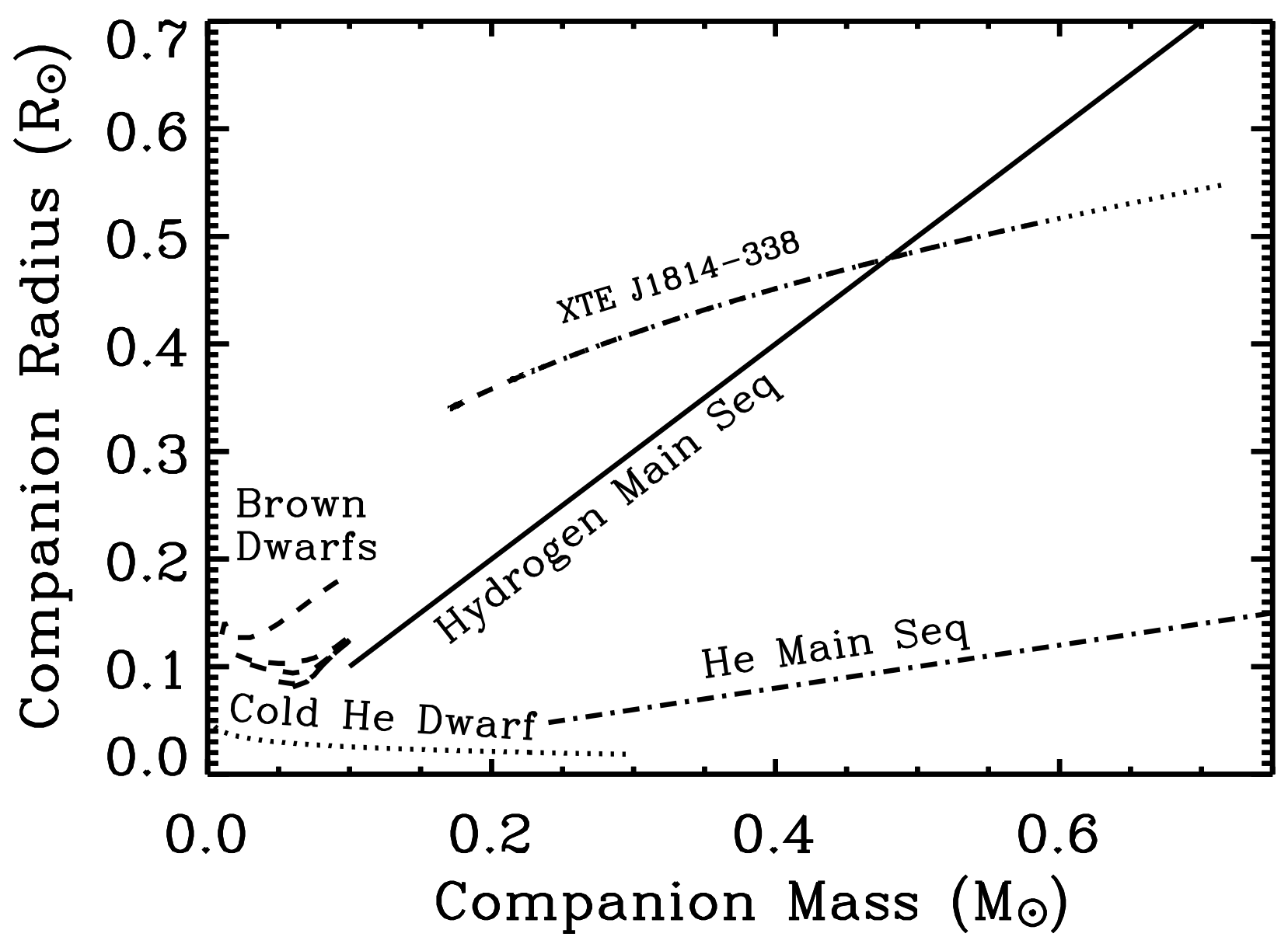

Fig. 14. - Companion mass vs. radius plane (similar to the Figure 4 of Markwardt et al. 2002): The lines under the phrase 'XTE J1814-338' are for the companion star in the source XTE J1814-338 (for an observer inclination angle $>20^{\circ}$ ); the dashed line is for a neutron star mass $1.4 M_{\odot}$ and the dotted line is for the neutron star mass $2.0 M_{\odot}$. For the purpose of comparison, we plot the mass-radius curves for hydrogen burning main sequence star (solid line; using $R / R_{\odot}=M / M_{\odot}$ ), helium main sequence star (dash-dot line; Verbunt 1990), brown dwarfs (dashed lines), and cold helium dwarf (dotted line). Brown dwarf models are for ages of $0.1,0.5,1,5$, and 10 billion years (from top to bottom). This figure demonstrates that the companion star in the source XTE J1814-338 is likely to be a hydrogen burning main sequence star. 
Table 1. Grid values considered for the parameters for likelihood calculations.

\begin{tabular}{cl}
\hline \hline Parameter & Grid values \\
\hline & $3.60,3.70,3.80,3.90,4.00,4.10$, \\
& $4.15,4.20,4.25,4.30,4.35,4.40$, \\
$R / M$ & $4.45,4.50,4.55,4.60,4.65,4.70$, \\
& $4.75,4.80,4.85,4.90,5.00,5.10$, \\
& $5.20,5.30,5.40,5.50,5.60,5.70$, \\
& $5.80,5.90,6.00$ \\
\hline \multirow{3}{*}{$i$} & $20^{\circ}, 22^{\circ}, 24^{\circ}, 26^{\circ}, 28^{\circ}, 30^{\circ}, 32^{\circ}$, \\
& $34^{\circ}, 36^{\circ}, 38^{\circ}, 40^{\circ}, 42^{\circ}, 44^{\circ}, 46^{\circ}$, \\
& $48^{\circ}, 50^{\circ}$ \\
\hline$\theta_{\mathrm{c}}$ & $10^{\circ}, 30^{\circ}, 50^{\circ}, 70^{\circ}, 90^{\circ}$, \\
& $110^{\circ}, 130^{\circ}, 150^{\circ}, 170^{\circ}$ \\
\hline$\Delta \theta$ & $5^{\circ}, 25^{\circ}, 45^{\circ}, 60^{\circ}$ \\
\hline \multirow{3}{*}{$n$} & $0.00,0.20,0.40,0.45,0.50$, \\
& $0.55,0.60,0.65,0.70,0.75$, \\
& $0.80,0.90,1.00,1.10,1.50$ \\
\hline
\end{tabular}


Table 2. $90 \%$ confidence intervals for $R / M, i$ and $n$.

\begin{tabular}{cccc}
\hline \hline \multirow{2}{*}{ Parameter } & EOS & Lower limit & Upper limit \\
\hline \multirow{2}{*}{$R / M$} & $\mathrm{~A} 18+\delta v+\mathrm{UIX}$ & 4.7 & 6.0 \\
\cline { 2 - 4 } & $\mathrm{A} 18$ & 4.2 & 6.0 \\
\hline \multirow{2}{*}{$i$} & $\mathrm{~A} 18+\delta v+\mathrm{UIX}$ & $26^{\circ}$ & $48^{\circ}$ \\
\cline { 2 - 4 } & $\mathrm{A} 18$ & $36^{\circ}$ & $50^{\circ}$ \\
\hline \multirow{2}{*}{$n$} & $\mathrm{~A} 18+\delta v+\mathrm{UIX}$ & 0.55 & 1.30 \\
\cline { 2 - 4 } & $\mathrm{A} 18$ & 0.55 & 1.17 \\
\hline
\end{tabular}


Table 3. $90 \%$ confidence intervals for the polar angle $\left(\theta_{c}\right)$ of the center of the spot.

\begin{tabular}{|c|c|c|c|c|}
\hline Parameter & EOS & Burst group & Lower limit ${ }^{a}$ & Upper limit $^{\mathrm{a}}$ \\
\hline \multirow{6}{*}{$\theta_{\mathrm{c}}$} & \multirow{3}{*}{$\mathrm{A} 18+\delta v+\mathrm{UIX}$} & Bursts: $1-8$ & $69^{\circ}$ & $131^{\circ}$ \\
\hline & & Bursts: $9-16$ & $82^{\circ}$ & $138^{\circ}$ \\
\hline & & Bursts: $17-22$ & $82^{\circ}$ & $139^{\circ}$ \\
\hline & \multirow{3}{*}{ A18 } & Bursts: $1-8$ & $60^{\circ}$ & $118^{\circ}$ \\
\hline & & Bursts: $9-16$ & $74^{\circ}$ & $129^{\circ}$ \\
\hline & & Bursts: $17-22$ & $69^{\circ}$ & $129^{\circ}$ \\
\hline
\end{tabular}

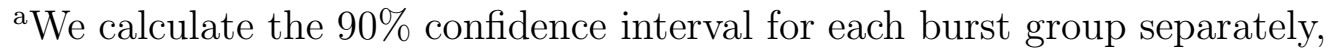
because this parameter could in principle vary between bursts. 
Table 4. Likelihood distribution of the angular radius of the spot $\Delta \theta$.

\begin{tabular}{|c|c|c|c|}
\hline Burst group ${ }^{\mathrm{a}}$ & Parameter & EOS: A18+ $\delta v+\mathrm{UIX}$ & EOS: A18 \\
\hline & $\Delta \theta$ & Likelihood & Likelihood \\
\hline & $5^{\circ}$ & 1.000 & 1.000 \\
\hline \multirow[t]{3}{*}{ Bursts: $1-8$} & $25^{\circ}$ & 0.566 & 0.426 \\
\hline & $45^{\circ}$ & 0.503 & 0.082 \\
\hline & $60^{\circ}$ & 0.047 & 0.002 \\
\hline \multirow{4}{*}{ Bursts: $9-16$} & $5^{\circ}$ & 0.718 & 0.826 \\
\hline & $25^{\circ}$ & 0.458 & 0.712 \\
\hline & $45^{\circ}$ & 1.000 & 1.000 \\
\hline & $60^{\circ}$ & 0.379 & 0.153 \\
\hline \multirow{4}{*}{ Bursts: $17-22$} & $5^{\circ}$ & 0.367 & 0.569 \\
\hline & $25^{\circ}$ & 0.439 & 0.649 \\
\hline & $45^{\circ}$ & 1.000 & 1.000 \\
\hline & $60^{\circ}$ & 0.354 & 0.110 \\
\hline
\end{tabular}

${ }^{a}$ We calculate likelihood distribution for each burst group separately, because this parameter could in principle vary between bursts. 